\title{
Effect of Finger Pressing on Anti-Inflammatory Effect in Obese Rats with Insulin Resistance
}

\author{
Shaotao Chen ${ }^{1}$, Lin Jiang ${ }^{2}$, Xiaolin Zhang ${ }^{1}$, Mingjun Liu ${ }^{1, *}$ \\ ${ }^{1}$ Changchun University of Traditional Chinese Medicine, Changchun, Jilin 130117, China \\ ${ }^{2}$ Balipu Community Health Service Center, Erdao District, Changchun, Jilin 130117, China \\ *Corresponding Author
}

Keywords: Finger pressure method, Insulin resistance, Obese rats

\begin{abstract}
Objective: To observe the anti-inflammatory effect of finger pressure method on obese rats with insulin resistance and study its mechanism. Methods: 20 male SD rats aged 5-8 weeks were randomly divided into two groups: general diet plus finger pressure method group and high fat plus finger pressure method group. They were fed with high fat diet for 8 weeks. The success of modeling was judged by fasting blood glucose, fasting insulin level and glucose tolerance test. The model of insulin resistance induced by obesity was induced by high fat diet for 8 weeks in the high fat plus finger pressure method group and the general diet plus finger pressure method group. After the experiment, blood glucose, blood insulin, TG, TCH, LDL and FFA were measured, and the fatbody ratio around testis and kidney was measured by killing animals. Results: The body weight, Lee index and fat coefficient of obese rats were significantly increased by high fat diet. Aerobic exercise and polysaccharide intervention can decrease the above morphological indexes. After 4 weeks of hypoxic exercise, the rats lost weight $(\mathrm{P}<0.05)$, impaired glucose tolerance, insulin resistance and insulin sensitivity. The basic finger pressure method intervention can alleviate the anti-inflammation and improve the insulin resistance of obese rats. Conclusion: Finger pressing can reduce blood lipid, control obesity and improve insulin sensitivity.
\end{abstract}

\section{Introduction}

Obesity is not only a metabolic disease, but also an anti-inflammatory disease of chronic obese rats. Obesity, lipid metabolism disorder, insulin resistance are closely related to the occurrence of type 2 diabetes and abnormal glucose tolerance. At present, many studies have shown that insulin resistance is the main pathogenesis of type 2 diabetes, and exercise training can improve insulin resistance. Recent studies have shown that finger pressure method has hypoglycemic effect, and some clinical cases have reported that it can be used for adjuvant treatment of type 2 diabetes [2]. In this study, the nutritional obesity rat model was induced by high fat diet, and the effects of finger pressure method and diet adjustment on serum tumor necrosis factor-and fasting insulin in nutritional obesity rats were studied. The effects of finger pressure method and diet adjustment on weight loss in obese rats were expounded from the point of view of the influence of finger pressure method on insulin resistance.

\section{Research Objects and Methods}

\subsection{Research Object}

20 SD male rats, aged 5-8 weeks. Ten rats were randomly selected as normal general diet plus finger pressure method group and fed with common feed. The remaining 10 rats were fed with high fat diet for 3 weeks, during which they were weighed regularly every week and their body length was measured. The ambient temperature is $20 \sim 24^{\circ} \mathrm{C}$, the relative humidity is $44 \% \sim 55 \%$, the rats are illuminated for $12 / 12 \mathrm{~h}$ day and night, weighed every other day, and the rats are observed daily (such as food consumption, water consumption, animal back hair, etc.). 


\subsection{Animal Grouping and Intervention Program}

Rats were randomly divided into two groups: general diet plus finger pressure method group and high fat plus finger pressure method group. The general diet plus finger pressure method group was given basic diet, while the high fat plus finger pressure method group was given high fat diet. The swimming program without weight bearing endurance was adopted. The swimming water temperature was $30.2^{\circ} \mathrm{C}$, the water depth was $50 \mathrm{~cm}$, and each rat had an active area of $200 \mathrm{~cm}^{2}$ to ensure the rats' continuous activities. The first week is the adaptive training stage, which starts from $10 \mathrm{~m} / \mathrm{min}$, increases by $5 \mathrm{~m} / \mathrm{min}$ at the speed of each stage, and each stage lasts for $15 \mathrm{~min}$ and gradually increases to the preset load. Since the second week, it is the formal experimental stage, and the treadmill training is carried out regularly according to the set exercise load every day. The weight changes observed every two weeks showed that the weight of rats in each group showed an upward trend after the experiment started. Before treatment (8th week), there was no significant difference in body weight between rats treated with finger pressure or resveratrol and obese rats. Once a day for 8 weeks. At the end of the experiment, rats were killed and blood samples, kidney, liver, perirenal fat and peritesticular fat tissues were collected for testing.

\subsection{Observation Index and Determination Method}

The rats were fasted for $12 \mathrm{~h}$, and the fasting blood glucose and fasting insulin were measured by fundus venous blood. $12 \%$ chloral hydrate $(0.4 \mathrm{~m} \mathrm{~L} / 100 \mathrm{~g}$ body weight $)$ was injected intraperitoneally for anesthesia, and blood was collected from abdominal aorta, centrifuged at $3^{\circ} \mathrm{C}$ for $15 \mathrm{~min}$ at $2000 \mathrm{r} / \mathrm{min}$, and supernatant was collected. Determination of serum free fatty acids by one-time colorimetric extraction. Serum insulin level was measured by radioimmunoassay, and insulin sensitivity index was calculated. The insulin resistance index was evaluated by steady-state model evaluation. After modeling, all rats were tested for Lee's index and fat body rate to analyze whether the modeling was successful. And Lee's index and fat body rate measurement are effective indexes to evaluate the obesity degree of adult rats.

\subsection{Statistical Analysis}

The statistical software SPSS 17. 0 was used for data statistics, and the data results were expressed as $(\bar{x} \pm \mathrm{s})$. The significant differences between groups were compared by variance analysis, and the significance level was set as $\mathrm{p}<0.05$.

\section{Result}

\subsection{Effect of Finger Pressure Method on Blood Glucose in Obese Rats}

The blood glucose changes observed every two weeks after the start of treatment showed that there was no significant difference in blood glucose between the rats in finger pressure method group and those in high fat diet plus finger pressure method group before treatment and those in obesity group. After treatment, fasting blood glucose of rats in general diet plus finger pressure method group and rats in high fat diet plus finger pressure method group showed a downward trend compared with obese group, while postprandial blood glucose had no significant difference compared with obese group. The ventromedial nucleus is full of the point activity of central nerve cells per unit time. The results show that finger pressure method can obviously reduce the excitability of lateral hypothalamus, increase the point activity frequency of ventromedial nucleus, inhibit the appetite of obese rats, reduce the intake of calories and achieve the purpose of losing weight. Compared with the high-fat diet group, the body mass and visceral fat mass of rats in the general diet plus finger pressure method group and the high-fat diet plus finger pressure method group decreased, and the difference was statistically significant.

\subsection{Effects of High-Fat Diet on Body Weight and Glucose Tolerance in Rats}

After 8 weeks of high-fat diet, the weight of rats in high-fat diet plus finger pressure method increased significantly, which was significantly different from that in normal diet $(\mathrm{P}<0.01)$. 
Glucose tolerance of rats in high fat diet plus finger pressure method group was significantly lower than that in general diet plus finger pressure method group. Compared with RD group, serum FFA, leptin, IL-1 and TNF- $\alpha$ in HFD group were significantly higher, and serum insulin level and HOMA-IR in HFD group were significantly higher, which indicated that high-fat feeding for 6 weeks could lead to insulin resistance in rats. The expression of SIRT1 in white adipose tissue of model rats was higher than that of obesity group after 8 weeks of finger pressure treatment $(\mathrm{P}<0.05)$. After 8 weeks of resveratrol intragastric treatment, the expression of SIRT1 in white adipose tissue of model rats was higher than that of normal diet group before modeling, and there was no significant difference $(\mathrm{P}>0.05)$. After 8 weeks of experimental intervention, it showed that the weight of rats in high fat diet group was significantly higher than that in normal diet group, with statistical significance $(\mathrm{P}<0.05)$.

\subsection{Comparative Analysis of Fat-Body Ratio of Rats in Each Group}

It can be seen from Table 1 that the ratio of fat body around testis, kidney and visceral fat body in the high fat plus finger pressure method group is higher than that in the general diet plus finger pressure method group $(\mathrm{P}<0.05)$. After finger pressure method intervention, the ratio of fat body around testis, kidney and viscera in middle and high doses was lower than that in high fat plus finger pressure method group $(\mathrm{P}<0.05)$, but there was no significant difference between general diet plus finger pressure method group and high fat plus finger pressure method group.

Table 1 Lipid-Body Ratio Around Testis and Kidney and Visceral Lipid-Body Ratio of Rats in Each Group ( $\bar{X} \pm \mathrm{s})$

\begin{tabular}{|l|l|l|l|l|}
\hline Group & $\mathrm{n}$ & $\begin{array}{l}\text { Lipid-body ratio around } \\
\text { testis(\%) }\end{array}$ & $\begin{array}{l}\text { Perirenal lipid-body } \\
\text { ratio(\%) }\end{array}$ & $\begin{array}{l}\text { Visceral fat-body } \\
\text { ratio(\%) }\end{array}$ \\
\hline $\begin{array}{l}\text { General diet plus finger pressure } \\
\text { method group }\end{array}$ & 10 & $1.33 \pm 0.21$ & $1.24 \pm 0.41$ & $2.55 \pm 0.67$ \\
\hline $\begin{array}{l}\text { High fat plus finger pressure } \\
\text { method group }\end{array}$ & 10 & $3.01 \pm 0.33$ & $1.84 \pm 0.64$ & $4.89 \pm 0.81$ \\
\hline
\end{tabular}

\subsection{Effect of Finger Pressure Method on Blood Glucose and Isi in Obese Rats}

Compared with the general diet plus finger pressure method group, Glu and Ins of rats in the high fat plus finger pressure method group increased significantly, ISI decreased significantly, but there was no significant difference in FruA. Glu and ISI in finger pressure method group and RTZ group were significantly lower than those in positive diet plus finger pressure method group, ISI was significantly increased, and fructosamine level had no significant difference (table 2).

Table 2 Effect of Finger Pressure Method on Blood Glucose and Isi in Obese Rats

\begin{tabular}{|l|l|l|l|l|}
\hline Group & Glu $(\mathrm{mmol} / \mathrm{L})$ & FruA $(\mathrm{mmol} / \mathrm{L})$ & Ins $(\mathrm{pmol} / \mathrm{L})$ & ISI \\
\hline General diet plus finger pressure method group & $6.33 \pm 0.32$ & $0.55 \pm 0.04$ & $93.04 \pm 20.14$ & $-5.81 \pm 0.31$ \\
\hline High fat plus finger pressure method group & $6.14 \pm 0.83$ & $0.64 \pm 0.21$ & $80.24 \pm 15.88$ & $-6.43 \pm 0.24$ \\
\hline
\end{tabular}

\section{Discussion}

Adipose tissue is also an important endocrine organ of human body, which can secrete many cytokines, such as FFA, leptin, TNF- $\alpha$, IL-1, resistin, adiponectin and visfatin, etc. The expression of adipocytes secreted by obesity increases or decreases due to the hypertrophy and increase of adipocytes. There are many causes of insulin resistance, such as obesity, oxidative stress, the internal mechanism of cells and the lack of trace elements. Among them, insulin resistance caused by obesity is characterized by impaired insulin function which promotes glucose uptake in adipose tissue and muscle. Effect of high fat diet on SIRT1 protein level; High-fat diet significantly reduced the expression of SIRT1, and inhibited the activity of pyruvate dehydrogenase, thus inhibiting the oxidation of glucose and the metabolism of human peripheral glucose. According to the theory of "islet-adipocyte axis" put forward by Hou et al, the body can form a feedback loop through insulin and leptin as information, thus regulating the balance of energy metabolism [3]. This study shows 
that finger pressure method can significantly control the increase of body weight and Lee's index caused by high-fat and high-sugar diet, and reduce the abdominal fat content represented by epididymal fat pad, which may be an important mechanism for improving insulin resistance by finger pressure method. In this experiment, IR rat model induced by obesity was established. IR caused by obesity has the characteristics of obesity and IR at the same time, that is, the weight and body fat of rats are increased, the insulin sensitivity index is decreased, and the fasting insulin level is increased.

After 8 weeks of feeding with high-fat diet, the body weight, blood triglyceride, free fatty acid and cholesterol of experimental rats were significantly higher than those of rats fed with common diet, suggesting that high-fat diet had a significant effect on lipid metabolism of experimental animals. Gong et al. in order to understand the role of the central nervous system in the pathogenesis of obesity and the effect of finger pressure method on the central nervous system of obese rats, after finger pressure method treatment, the content of 5-HT and 5-t3T/5-Hl.nn in the raphe nucleus of the brain stem of obese rats increased, and the metabolic rate increased, which may inhibit the feeding activity of rats by affecting the satiety center function [4]. In recent years, Zhang and other research groups found that blood fatty acids (which are transported to muscles during exercise) can mobilize more triglycerides and triglyceride-rich lipoproteins for further hydrolysis, thus achieving the purpose of regulating blood lipids and reducing body fat content [5]. The weight gain of rats in the general diet plus finger pressure method group and the high fat plus finger pressure method group through the intervention of finger pressure method method is slow, which shows that the combined intervention of exercise and finger pressure method method has a certain effect on weight control. The intervention of SIRT1 specific agonist resveratrol on NF- $\kappa \mathrm{B}$ gene expression in white adipose tissue of model rats in this study is more obvious than that of finger pressure method therapy, suggesting that SIRT1 has intervention effect on NF- $\kappa \mathrm{B}$ gene expression in white adipose tissue of IR obese rats [6]. In this study, the levels of serum adipokines in rats fed with high fat for a long time were significantly increased, and the levels of FFA, leptin and TNF- $\alpha$ were positively correlated with IR, which confirmed that IR induced by high fat diet might be realized by increasing the levels of adipokines.

The results of this study showed that the body weight and body fat/body weight ratio of rats after finger pressure method intervention were lower than those of model group, which indicated that the insulin resistance degree of high-fat diet plus finger pressure method group increased obviously, but insulin sensitivity decreased, reflecting the insulin resistance state of high-fat diet plus finger pressure method group. There is a certain leptin receptor in islet B cells, and leptin can directly act on its receptor to inhibit insulin secretion, thus alleviating hyperinsulinemia. At the same time, the serum Ins level was significantly lower than that of P general diet plus finger pressure method group, and ISI was significantly higher, which indicated that finger pressure method could improve insulin sensitivity and insulin resistance. PDX-1 can regulate the development of islet $\beta$ cell population [7-8], GK is a sensor of insulin secretion, which can directly or indirectly regulate blood glucose concentration [9]. Therefore, hyperinsulinemia is also one of the manifestations of IR in the body, and the degree of IR in the body can be measured by measuring the fasting plasma insulin concentration [7]. Suo et al. experimental studies show that for obese rats with obvious clinical characteristics, finger pressure method can reverse leptin in the center and periphery, and correct leptin resistance and insulin resistance [10]. However, the IR obesity model used in this study is similar to human epidemiology, with high reliability and stable expression of traits. The observation and record of metabolic related indexes showed that IR obese rat model was successfully established by feeding high fat diet continuously for 8 weeks.

This study found that the weight of rats decreased significantly after 6 weeks of intervention with polysaccharide, but the Lee index and fat coefficient decreased without statistical significance It is found that long-term aerobic exercise can improve the anti-inflammation of obese rats with adipose tissue, increase the size and irregularity of adipose tissue cells, and inhibit the infiltration of macrophages. In visceral obesity, basal and postprandial hyperinsulinemia exists, which downregulates insulin receptor gene through negative feedback mechanism, reduces the synthesis of 
insulin receptor protein and the combination of insulin and receptor, and hinders insulin signal transduction; Du et al.' s research on ZDF mice shows that exercise can improve glucose uptake by skeletal muscle stimulated by insulin, but can not affect insulin receptor signal transduction through phosphatidylinositol 3 kinase pathway [11]. The effect of finger pressure method therapy on TNF- $\alpha$ protein level in IR obesity model rats: After 8 weeks of finger pressure method therapy, the expression of TNF- $\alpha$ in white adipose tissue of rats in general diet plus finger pressure method therapy group was lower than that in obese group, which confirmed that obesity induced by high fat diet was closely related to anti-inflammatory factors of obese rats in adipose tissue, and further proved that obesity was one of the influencing factors that induced the expression of antiinflammatory factors in obese rats in adipose tissue. Therefore, in this experiment, the IR rat model was reproduced by feeding scientifically prepared high fat diet, and the feasibility of this method was evaluated by relevant indexes.

\section{Conclusion}

High fat diet can increase the body weight and lee's index of rats, activate anti-inflammatory factors and induce inflammatory reaction in obese rats. The results of this study suggest that finger pressure method can improve insulin sensitivity and effectively interfere with the occurrence and development of IR, and its mechanism may be related to its ability to reduce serum TG, GLU and serum free fatty acid levels and alleviate hyperinsulinemia. In contrast, finger pressure method can improve insulin sensitivity, control weight gain, reduce abdominal fat and reduce blood lipid. In the future, we need to combine more cell experiments and animal intervention experiments to confirm the above viewpoints.

\section{Acknowledgment}

Changbai Mountain through the adjustment of dirty techniques school heritage studio construction project. Item No.: LPGZS2019-11; National Natural Science Foundation of China-The Research of mechanism and Interventional effect on insulin resistance obesity patients with YunFuTongJing Manipulation. No.:81674092

\section{References}

[1] Yang Yubin, Ke Bin, Qin Jian. Effects of different calorie restriction methods on glucose and lipid metabolism and adipocyte differentiation in obese rats with insulin resistance. Chinese General Practice, No. 18, pp. 2156-2161, 2016.

[2] Zhang Xu, Zhou Shan, Chen Huizhen, et al. Establishment and evaluation of an obese rat model of liver depression and spleen deficiency insulin resistance. Chinese Journal of Basic Medicine in Traditional Chinese Medicine, Vol. 024, No. 010, pp. 1373-1376,1431, 2018.

[3] Hou Liqiong Zhao Tieyun Zhang Yiyi. Interventional study of berberine on insulin resistance in skeletal muscle of obese insulin resistant rats. Journal of Sichuan University: Medical Edition, No. 46, pp. 831, 2015.

[4] Gong Meirong, Chen Fengli, Cao Chen, et al. Study on electroacupuncture to improve insulin resistance and mitochondrial function in obese rats through AMPK/PGC-1 signaling pathway. Shizheng Guo Medicine, No. 05, pp. 235-237, 2017.

[5] Zhang He, Zhou Yue, Zhang Yimin, et al. Effect of hypoxic exercise on insulin resistance and blood lipid metabolism in obese rats. Journal of Beijing Sport University, Vol. 039, No. 009, pp. 44-49, 2016.

[6] Yang Hui, Zhou Yuanda, He Haixia. The effect of chlorogenic acid on insulin resistance in obese rats. Chongqing Medical, No. 018, pp. 2457-2460, 2015. 
[7] Yang Yubin, Ke Bin, Qin Jian. Effect of Jianpi Huatan Recipe on glucose and lipid metabolism in obese insulin resistant rats. Chinese Patent Medicine, Vol.40, No. 08, pp. 1822-1826, 2018.

[8] Liu Xia, He Junfeng, Qu Yating, et al. Electroacupuncture on insulin resistance in simple obese rats and the effects of hypothalamic agouti gene-related protein and neuropeptide Y. China Journal of Traditional Chinese Medicine Information, Vol.23, No. 05, pp. 62-65, 2016.

[9] Suo Linna, Wang Wei. The effect of liraglutide on serum omentin-1 and insulin resistance in obese rats induced by high-fat diet. Journal of China Medical University, Vol. 44, No. 12, pp. 79-81, 2015.

[10] Suolinna, Wang Wei. The effect of liraglutide on serum omentin-1 and insulin resistance in obese rats induced by high-fat diet. Journal of China Medical University, No. 012, pp. 1129 -1131, 2015.

[11] Du Juan, Zhu Anfeng, Song Dongming, et al. Morphological changes and significance of obese rats induced by high-fat diet. Chinese Journal of Endocrinology Surgery, Vol. 13, No. 6, pp. 463465, 2019. 